desde la academia

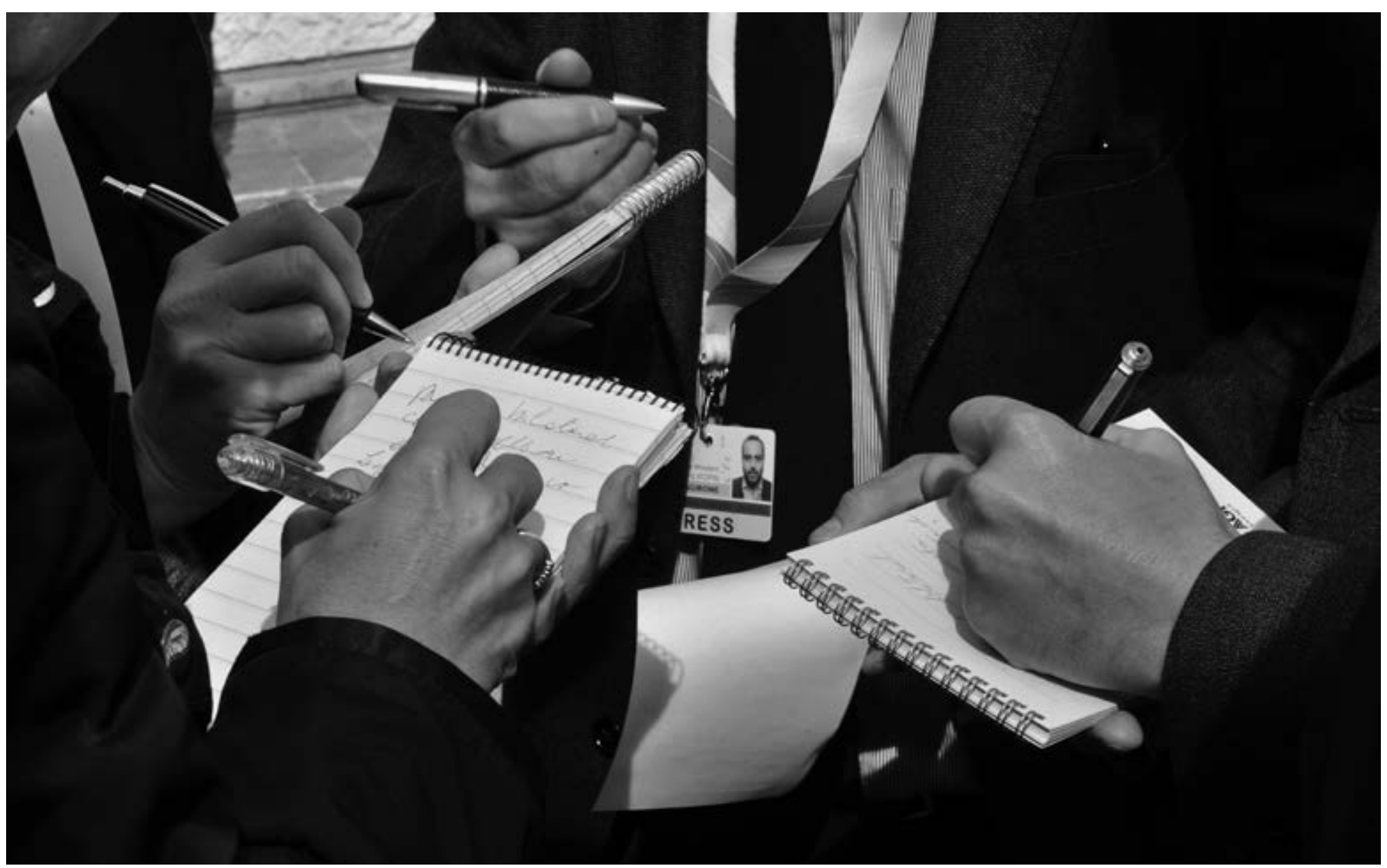

Foto: ๑AFP / Georges Gobet. Godollo, abril de 2011

\title{
Periodismo de datos en Uruguay
}

\section{Data Journalism in Uruguay}

https://doi.org/10.22235/d.v0i24.1167

Leandro Rodríguez 
Leandro Rodríguez Ege Üniversitesi [Universidad del Egeo] Esmirna, Turquia leandro8@adinet.com.uy

Recepción: marzo de 2016 Aceptación: mayo de 2016

Artículo traducido del inglés al español por Raquel Domínguez

\section{RESUMEN}

El término periodismo de datos es un término nuevo que llegó a las redacciones hace algunos años, pero ahora ningún medio quiere quedar afuera. En un país pequeño como Uruguay -a pesar de que en todas las redacciones están al tanto de su existencia y ha habido algunas iniciativas- es una práctica que en el año 2015 todavía no ha encontrado su lugar en los medios. Este trabajo busca esclarecer de qué se habla cuando se habla de periodismo de datos para luego, a través de información recabada de medios, organizaciones no gubernamentales y centros educativos, averiguar cuál es su situación actual en el país, los impulsos que ha tenido, los obstáculos que ha encontrado y el futuro que le espera.

Palabras clave: nuevos medios, periodismo digital, periodismo en Uruguay, periodismo de datos, periodismo científico

\section{Introducción}

Las nuevas tecnologías obligan a los medios de comunicación a reinventarse y a encontrar modos distintos de sobrevivir. Primero fue la radio, luego la televisión y ahora es Internet y las redes sociales. A través de los años, el periodismo ha evolucionado para continuar siendo un negocio rentable y así mantener su papel en el mundo moderno de las democracias desarrolladas; una postura en la que su independencia de los vínculos políticos y estatales lo han hecho más dependiente del sector privado y del impacto público que genera.

Hace diez años los diarios discutían si era o no posible el periodismo en línea, si era un trabajo redituable o una pérdida de tiempo. Hoy el periodismo en la red es un hecho, los modelos de negocio están claramente definidos y se ha avanzado mucho hacia la convergencia de me-

\section{ABSTRACT}

The term "data journalism" arrived to newsrooms some time ago, and now every news media wants be part of it. In a small country like Uruguay -despite the isolated efforts of some companies, NGOs and government agencies- it has still not found its place in the media. This research tries to clarify the concept of data journalism in order to break down the state of the art in Uruguay, through interviews with journalists, members of $\mathrm{NGO}$ s and members of the academic staff of the universities where the future journalists are being formed. Thus being able to point out the impulses and obstacles data journalism has encountered in order to foresee its possible future.

Keywords: new media, digital journalism, journalism in Uruguay, scientific journalism, data journalism

dios. De hecho, no solamente los diarios han entrado en el negocio del periodismo en Internet, sino que la mayoría de los canales de televisión actualmente compiten cabeza a cabeza con la industria gráfica, las emisoras de radio y los portales de noticias para ver quién consigue más tráfico en sus páginas.

Asimismo, se observa que el periodismo sigue cambiando, tanto en su estilo como en su contenido y en el proceso de narrar las noticias. Sirve como ejemplo la discusión que tiene lugar actualmente en la mayoría de las redacciones, sobre cuáles son los conocimientos que debe tener un periodista. ¿Es necesario que sepa de fotografía? ¿Debe saber de redes sociales? ¿Debe saber usar un micrófono profesional? ¿0 alcanza con que consiga información de valor y sea capaz de comunicarla? 
En medio de ese debate y durante este proceso de evolución ininterrumpida del periodismo, surgió lo que se dio en llamar periodismo de datos. Aunque nació de los avances tecnológicos que lo posibilitaron, llegó a un mundo en crisis. Un mundo lleno de programadores, periodistas, diseñadores, economistas e investigadores, pero carente del dinero necesario para sostener una actividad como el periodismo de datos. Algo que -como los portales web de noticias hace algún tiempo- era el sueño de todo periodista, pero la pesadilla de los directores de los medios.

El diario británico The Guardian inició en 2006 la primera gran ola del llamado periodismo de datos, al crear un blog dedicado a ese tema cuando se revelaron los archivos de WikiLeaks. En ese momento, se hizo evidente la necesidad de nuevas técnicas para elaborar las noticias, ya no a partir de la escasez de información, como era habitual, sino de su abundancia. Después de eso, todos los grandes diarios del mundo intentaron aproximarse al periodismo de datos, pero esa aproximación todavía está en un "período de prueba". 0, como dice Dan Ariely, profesor de Economía del Comportamiento, el periodismo de datos es como las relaciones sexuales en la adolescencia: todos hablan de eso, pero nadie lo hace (citado en Träsel, 2014).

En especial es difícil para los medios pequeños -y los mercados pequeños- prever un futuro en el que haya equipos dedicados exclusivamente al periodismo de datos, porque implica un esfuerzo económico y, además, un riesgo. Por eso es importante analizar qué es lo que está sucediendo con el periodismo de datos en un país como Uruguay, donde ni siquiera los medios privados de alcance nacional disponen de grandes presupuestos. El presente trabajo tiene como objetivo llamar la atención sobre este nuevo fenómeno y mostrar los obstáculos que encuentran las redacciones uruguayas para desarrollar este nuevo tipo de periodismo.

\section{Metodología}

Este trabajo se centra en el periodismo de datos en el Uruguay de hoy, por lo que se usaron técnicas cuantitativas y cualitativas para recoger los datos. Como los medios de alcance nacional no son muy grandes, fue posible preguntar directamente en los medios sobre su situación, sus opiniones y su trabajo, mediante entrevistas a sus directores.

El tener acceso a las personas directamente involucradas le quita relevancia al análisis de contenido como técnica para detectar los pros y los contras de cómo se está llevando a cabo el periodismo de datos en Uruguay. Como se verá más adelante, el periodismo de datos es un proceso y, si se intenta analizar ejemplos concretos, se debe inferir todo el proceso previo de elaboración a partir de esos artículos. Sin embargo, hablar con las personas que hacen esas historias proporciona un panorama más rico sobre cómo se realizaron. El testimonio de los implicados da una visión más clara del proceso. Por eso se eligió a la entrevista como técnica para describir los últimos avances de ese proceso en Uruguay.

Se realizaron entrevistas a algunas fuentes calificadas, previamente seleccionadas, por medio de un cuestionario cerrado que surgió de la conceptualización del término periodismo de datos. Las personas entrevistadas provienen de medios de comunicación privados, de organizaciones gubernamentales y no gubernamentales, y también de centros de enseñanza del periodismo (universidades). De esta manera se abarcó no solamente el punto de vista de los elaboradores de historias, 
sino que también se amplió el espectro para alcanzar más testimonios que esclarecieran cómo se considera al periodismo de datos en el país.

\section{¿Qué es el periodismo de datos?}

Muchas personas trabajan actualmente en el área de periodismo de datos, existen premios para piezas de periodismo de datos, libros sobre este género, charlas, eventos y demás. Pero ante la interrogante sobre qué es el periodismo de datos, la mayoría de los periodistas y especialistas terminan respondiendo con definiciones vagas, o comienzan a enumerar lo que no es, o cuáles son algunas de sus características.

Existen pocas aproximaciones a una definición clara y académica de su significado, y esto es un problema, porque hasta que no se haya definido de qué estamos hablando, no vamos a saber qué estamos diciendo.

En el Manual del periodismo de datos (Gray, Bounegru y Chambers, 2012) hay un apartado que se titula "¿Qué es el periodismo de datos?" pero, a la hora de dar una definición concreta, el autor admite que decir que es "periodismo elaborado con datos" no sirve de mucho. Y como no sirve de mucho, comienza a aportar algunos conceptos básicos, las características del género y algunos ejemplos. Así que, al fin y al cabo, no hay definición. Pero sí menciona algo presente en todas las descripciones del periodismo de datos: es un proceso.

Por ejemplo, el periodista Mirko Lorenz define el periodismo sostenido por datos como un plan de trabajo progresivo que consiste en encontrar, procesar, visualizar y elaborar una historia a partir de cantidades "significativas" de datos (Lorenz, 2010).
El término significativo es muy ambiguo, porque podría referirse a grandes cantidades de datos, como las bases de datos. Pero estas bases no son la única fuente para este tipo de flujo de trabajo, ya que en el caso de WikiLeaks, por ejemplo, se trató de una filtración de documentos confidenciales de Estados Unidos a la prensa. Para evitar esta ambigüedad, diré que las fuentes usadas por el periodismo de datos son tan ricas en información que la información misma es un obstáculo para la relevancia. Los datos se convierten a la vez en ruido y oro en el periodismo de datos, de modo que la tarea es identificar lo que es noticia y esconder el ruido. Y lo más interesante es que una porción de información puede ser al mismo tiempo las dos cosas, según cómo se cuente la historia.

La cuestión no reside en la cantidad de archivos Excel, en los PDF o en una buena cantidad de documentos. Si todos los archivos de WikiLeaks hubieran sido relevantes no habría sido necesario analizarlos, pero como la mayoría de ellos no tenían valor para el público en general, fue preciso usar un "filtro" y se necesitó del plan de trabajo progresivo del periodismo de datos para que esos documentos se transformaran en noticia. De modo que la tarea de este periodismo es organizar los datos de tal manera que surja de ellos una historia. Uso el término organizar y no seleccionar o filtrar porque a veces los periodistas son los que eligen los datos relevantes, pero otras veces dejan esa decisión en manos del lector.

Se toma como ejemplo el caso de Siete mil millones... y usted, ¿sabe qué número es?, una aplicación creada por la British Broadcasting Corporation (BBC) cuando las Naciones Unidas anunciaron que la población mundial había alcanzado esa cifra (BBC, 2011). Aquí la noticia no consistía en buscar la relación entre los datos 


\section{Search Results: 5160 files found}

\section{Health Download Name}

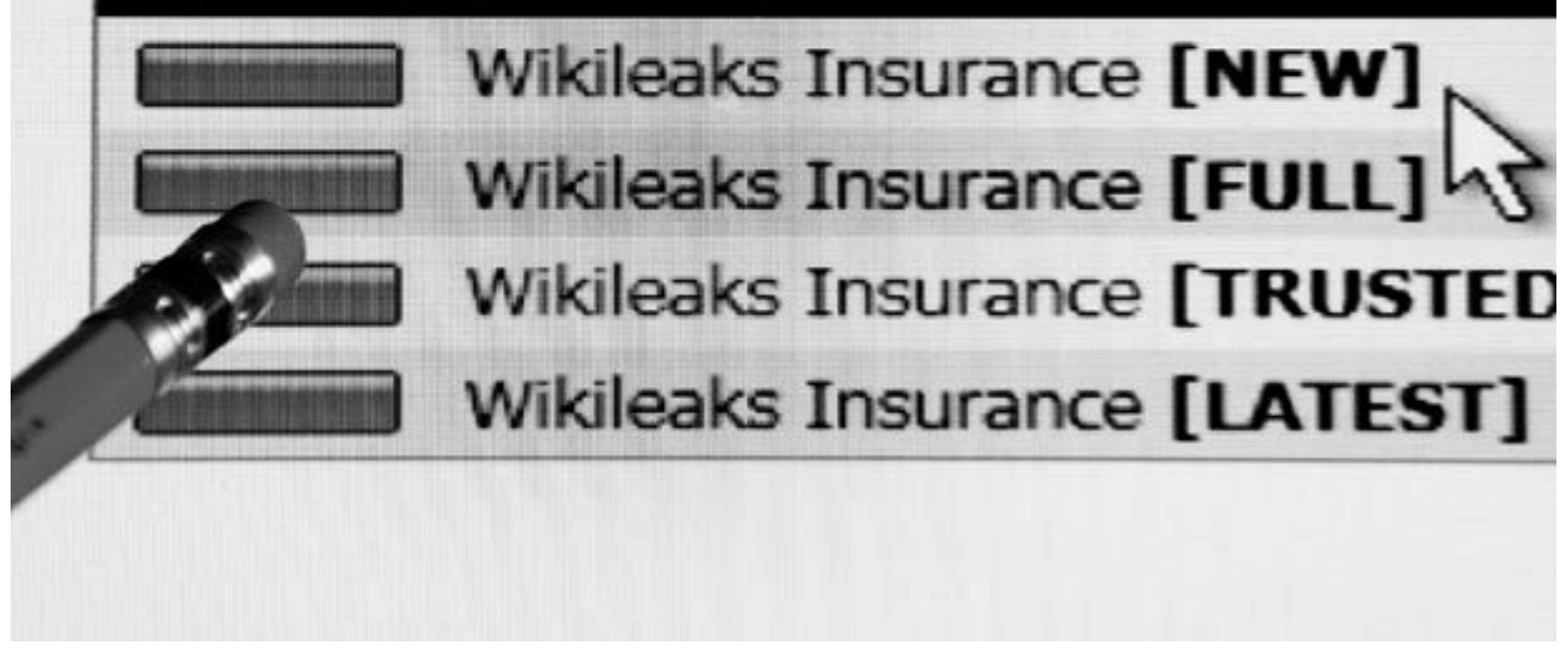

obtenidos, sino en dejar que el lector la armara a su propia manera apoyándose en los datos que se desprendían de la celebración de ese hito. En este caso, la historia fue elaborada tanto por la información pública como por la personal. Y lo que para un usuario es una información relevante podía no serlo para los demás. Esta es la mejor demostración de la personalización en el periodismo. No se trataba de filtrar o limpiar los datos, sino de organizarlos de manera que el lector fuera el encargado de darle relevancia a la información.

Pero es necesario hacer notar dos cosas más. En primer término, si no hubiera noticia, no tendría sentido alguno elaborar un artículo interactivo. No sería periodismo, sino un juego estadístico, una página recreativa en la red. Es la existencia de una noticia lo que justifica crear la aplicación interactiva. En segundo término, si en vez de hacer eso hubieran publicado las hojas de cálculo de las Naciones Unidas, tampoco habría sido periodismo de datos. Así que ahora hay dos factores más que resultan decisivos en el proceso: la existencia de una noticia y la visualización.

En un mundo cada vez más visual, en una sociedad en donde todo debe ser comprendido velozmente, se precisa de la visualización para producir un rápido impacto sobre el público y para transformar los números en símbolos familiares: imágenes. Pero es un tema a discutir si la visualización es una condición indispensable para el periodismo de datos. ¿Qué pasa si se descubre en las actas oficiales que la Justicia juzga de modo diferente según la raza o el origen étnico de los acusados, como lo descubrieron los periodistas estadounidenses Donald Barlett y James Steele en 1973? (Dader y Gómez, 1993) ¿Eso no es periodismo de datos? ¿Cómo puede la visualización de los datos hacer la diferencia en esa noticia?
Foto: ${ }^{\circ}$ AFP / Paul J. Richards. Washington, DC, diciembre de 2010 
En este trabajo se defiende como punto de vista que la visualización, si bien no forma la parte central de la noticia en la mayoría de los casos, es un nuevo medio para comunicar los datos de manera que el público pueda entenderlos. De modo que, aunque no sea una condición necesaria para elaborar una historia de periodismo de datos, es una herramienta muy poderosa que no puede ser usada en otro tipo de noticias como se utiliza en este género.

Tomando en consideración todos los conceptos mencionados, se puede elaborar la siguiente definición: El periodismo de datos es el proceso de elaborar una noticia a partir de una fuente cuyos datos son tan abundantes que se transforman en ruido, y por lo tanto se hace necesario organizar esos datos para extraer una historia y poder comunicarla.

Esta definición tiene aún dos conceptos vagos: datos y abundante. Entonces, ¿qué significa, exactamente, datos, y en qué punto se hacen tan abundantes que se transforman en ruido? La palabra datos, en este contexto, se usa desde un punto de vista periodístico. Los datos, entonces, son solo las piezas de información que tienen la potencialidad de justificar la producción de texto en los medios. Por tanto, los datos contienen, en primer lugar, un factor ignorado o escondido que obliga a una hipótesis. Allí puede haber algo, pero no se sabe qué es hasta que se ven esos datos con más detalle. Y, en segundo lugar, existe un proceso de elaboración de las noticias, de modo que hay una persona que está buscando la relevancia en los datos a fin de componer una historia. A esto se asocia la cuestión de qué son las "noticias" y cuándo se justifica hacer una historia en los medios masivos de comunicación, pero ese tema escapa al propósito de este artículo y ha sido largamente discutido por varios autores.
En relación con la abundancia de los datos, no existe un punto preciso en el cual la información deja de ser tal para convertirse en ruido. Si bien es una cuestión de cantidad, también es una cuestión de recursos humanos. Digamos que, por ejemplo, a partir de una entrevista de una hora de duración los datos producidos pueden ser manejados fácilmente: se pueden manejar las citas, se puede añadir contexto y hasta se puede publicar la entrevista completa. Pensemos ahora en una serie de diez entrevistas de una hora de duración cada una. Esta cantidad de información todavía puede ser manejada por un periodista, pero también se pueden aplicar otros métodos antes que los tradicionales del periodismo. Se puede hacer un análisis cuantitativo para extraer más información o para resumir lo que se dijo. Pero eso no debe llevar a pensar que el periodismo de datos se trata solamente de métodos cuantitativos. En este caso, tanto los métodos periodísticos como los de investigación pueden ser usados para confeccionar la noticia. Por lo tanto, no existe una frontera que separe al ruido de los datos relevantes, sino un punto de vista y una técnica en el proceso de elaboración de las noticias. En este caso es posible usar tanto el periodismo de datos como el tradicional.

Supongamos ahora un caso extremo: un archivo con 1500 entrevistas de una hora cada una. Aquí el periodismo tradicional no puede hacer nada, y es cuando necesita la asistencia de la tecnología o la ayuda de métodos estadísticos y de investigación (que también requieren de la tecnología). No hay otro camino que confiar en la suerte para elaborar una noticia a partir de eso: extraer al azar y ver si allí hay una información relevante. Entonces para elaborar una noticia se precisa o bien una investigación cuantitativa o bien el trabajo de una cantidad de personas que ningún medio 
podría pagar. Sin embargo, un grupo numeroso de voluntarios, reunidos espontáneamente, podría manejar esta situación. Aquí es cuando Internet, su espíritu comunitario y los métodos de crowdsourcing se presentan como la clave del periodismo de datos. Eso es lo que sucedió cuando se publicaron los archivos de WikiLeaks. Es un caso típico de periodismo de datos y muestra por qué el desarrollo tecnológico fue un factor clave para promover este modo de trabajo en las redacciones de los diarios.

El periodismo de datos no puede entonces imaginarse de otro modo que no sea como un proceso. Y, visto como tal, se pueden distinguir cuatro pasos:

- Recolectar los datos: Es el primer paso, encontrar una base de datos, pedir información en un organismo público, analizar los buscadores, contratar hackers ${ }^{1}$, etcétera.

- Analizar los datos: Es el acto de descubrir la historia detrás de los datos. Puede suceder que la historia ya sea conocida, pero es preciso conseguir los datos que respalden esa hipótesis.

- Organizar los datos: Cuando se tienen los datos a disposición y la historia es clara, es necesario reacomodarlos para que los lectores los entiendan. Este es el momento de decidir: ¿La historia es única o hay múltiples historias? ¿Fue generada por un usuario? ¿Se va a mostrar en un artículo, una aplicación, un gráfico o una página web?

- Comunicar la historia: Una vez definida la historia y decidido el formato, debe elaborarse el producto final. Esta es la fase final, en la que el proceso se materializa en un producto concreto.
En este proceso, la visualización participa del tercer y cuarto paso, pero esto no quiere decir que deba participar necesariamente. Solo tiene lugar si existe una buena manera de organizar los datos para comunicarlos. Entonces, se la realiza durante la fase de elaboración del producto final. Debe observarse también que ningún paso requiere determinada especialidad, solamente la capacidad de elaborar una historia. Una sola persona puede hacer periodismo de datos tanto como un equipo formado por periodistas, programadores, hackers, especialistas en estadística y diseñadores de infografías. Disponer de un equipo puede dar como resultado un producto de mayor profundidad, porque la suma de capacidades específicas facilita el proceso, pero también puede terminar en una pérdida de tiempo, con la posible recolección de datos que no llevan a una noticia o la creación de gráficos donde no se los precisa.

Como se puede notar, los términos periodismo de datos, periodismo basado en datos y ejemplos del periodismo de precisión se utilizan indistintamente para construir la definición del género. Esto sucede porque, aunque se intente diferenciarlos, son diferentes caras de un mismo fenómeno que a través de los años, gracias al avance tanto de los conceptos teóricos como de las innovaciones tecnológicas, ha culminado en lo que hoy se denomina periodismo de datos. Prueba de esto son los acontecimientos que se enumerarán a continuación.

\section{Una breve historia}

La primera pieza de periodismo de datos, o por lo menos así lo considera el propio medio, fue publicada por el diario británico The Guardian en 1821 (Rogers, 2011). Aquel artículo no tenía nada especial, salvo el uso de una tabla que mostraba la cantidad de alumnos que concurrían a las escuelas de Manchester y Salford en ese momento, y el presupuesto que se invertía en ellos.
$1::$

La palabra hacker designa a las personas que aprovechan las debilidades de una red de computadoras o un sistema informático, con propósitos diversos. A medida que los hackers se hicieron famosos por sus acciones, a fines de los 90 y en la primera década de este siglo, también se comenzó a usar para describir una filosofía, la de la cultura informática alternativa, muchas veces también asociada a actividades ilegales o éticamente cuestionables (Yagoda, 2014). 


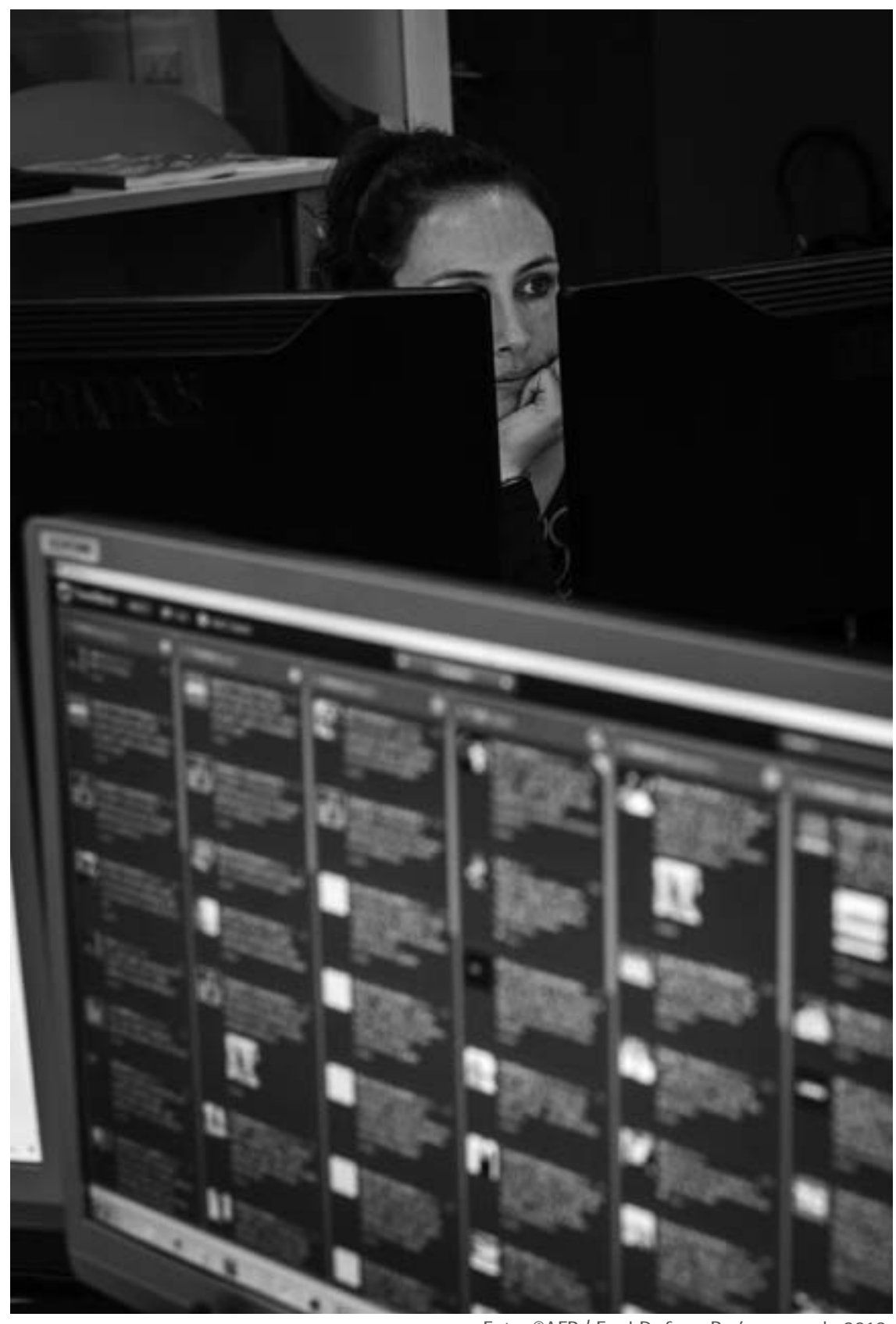

Foto: ${ }^{\circledR A F P}$ / Fred Dufour. Paris, enero de 2013
De acuerdo a un artículo publicado por ese mismo diario en 2011, la noticia fue chocante porque contradecía las estimaciones oficiales. Dejando la historia a un lado, fue la primera vez que un grupo grande de datos fue analizado por periodistas para conseguir una historia. Por supuesto que, comparado con el periodismo de datos actual, aquello fue simplemente la publicación de una hoja de cálculo. Pero en aquella época fue un modo diferente de elaborar noticias, un nuevo enfoque sobre cómo contar una historia.

Después de ese artículo, otras historias con uso de estadísticas fueron apareciendo, aisladamente, a través de los años, y en ellas fueron utilizadas nuevas técnicas de visualización. Pero debieron pasar 130 años para que volviera a surgir algo parecido al periodismo de datos.

Cuando se inventaron las computadoras nació una nueva manera de analizar los datos y, con ella, una nueva elaboración de las noticias: se llamó "reportaje asistido por computadora" o CAR, por sus siglas en inglés (Kuutti \&t Heikki, 2015). Lo que se hizo en esa época no se compara con el periodismo de datos actual, pero la invención de la nueva tecnología es un mojón importante en su historia. El periodismo que se practica hoy no se podría hacer sin computadoras, y está claro que el periodismo de datos actual no sería factible sin las posibilidades que la tecnología ofrece. En concreto, el periodismo de datos es el producto de la irrupción de nuevas tecnologías en las redacciones.

Mientras comenzaba a desarrollarse el CAR, nacía también un nuevo tipo de periodismo. No en el sentido tecnológico, sino conceptual. A fines de los cincuenta y durante la década del sesenta, Philip Meyer comenzó a usar técnicas de las ciencias sociales para recoger datos y elaborar 
historias a partir de ellos. Esto se conceptualizó unos años más tarde bajo el nombre de periodismo de precisión, en un libro con ese mismo nombre que se publicó en 1973 (Dader y Gómez, 1993). De hecho, fueron precisamente Meyer y sus colegas del nuevo periodismo científico los primeros en usar sistemáticamente el reportaje asistido por computadora en esos años (Coddington, 2015).

En la reedición del libro, que tituló El nuevo periodismo de precisión, Meyer (1991) define al género como "la aplicación de los métodos de investigación de las ciencias sociales y del comportamiento a la práctica del periodismo":

El nuevo periodismo de precisión es periodismo científico. (En Francia, el término "periodismo de precisión" ha sido traducido como "le journalisme scientifique"). Significa tratar al periodismo como si fuera una ciencia, adoptando el método, la objetividad y los ideales propios de la ciencia a todo el proceso de la comunicación para las masas (Meyer, 1991).

Aunque el periodismo de precisión fue bien recibido y siguió desarrollándose durante años, fue recién cuando irrumpieron las computadoras personales en los años noventa cuando se lo consideró un modo económicamente posible de elaborar una historia. Y llegado ese momento -como sucedió después con el periodismo de datos- ya no eran los periodistas los que recogían los datos, como sostenía Meyer. Los periodistas comenzaron a usar el CAR más a menudo como una manera de procesar los datos para entenderlos de un modo que, sin las nuevas tecnologías, era virtualmente posible pero pragmáticamente imposible.
El periodista no tomaba las técnicas de recolección de datos de las ciencias sociales para elaborar una historia, sino para analizarla. Considerando que una investigación social exige semanas o meses, hay pocos medios que puedan o quieran pagarla. Pero si otra persona elabora esa información y los periodistas la analizan, pasa a ser más factible. Y así se pasó del periodismo de precisión al periodismo de datos actual, donde el problema no es construir los datos - puesto que eso lo hacen no solamente los investigadores sociales, sino máquinas, instituciones, etcéterasino identificar su relevancia.

El uso de las PC se hizo habitual en las redacciones, nacieron los portales digitales de noticias y el periodismo se lanzó a buscar nuevas maneras de elaborar las historias. Tanto el periodismo de precisión y el reportaje asistido por computadora tomaron impulso como soluciones para la creciente cantidad de datos a disposición en el mundo digital. Fue en ese contexto que una organización, cuyo líder era Julian Assange, se dio a conocer en el 2010 al difundir varios documentos filtrados del gobierno estadounidense y ponerlos a disposición de un selecto grupo de diarios de distintos países.

En ese momento el periodismo de datos dejó de ser una sofisticada novedad para convertirse en una verdadera necesidad en las salas de redacción de todo el mundo. Los documentos filtrados por WikiLeaks eran tan numerosos que surgió una súbita necesidad de habilidades y destrezas que no estaban presentes en los medios, para extraer historias de los informes gubernamentales secretos. Como señala el Manual iberoamericano de periodismo de datos, WikiLeaks tenía una gran cantidad de información, pero necesitaba la ayuda de periodistas para comprobar las fuentes de los 
datos, escribir las historias y adquirir credibilidad para atraer la atención del público (Perry y Paz, 2014). En otras palabras, tenían una gran cantidad de datos, pero precisaban la ayuda de periodistas para hacerlos valer.

The Guardian, uno de los diarios que colaboraba con WikiLeaks, inició en ese momento el Datablog ${ }^{2}$, un blog dedicado al periodismo de datos donde quedaba claro que no se trataba de algo excepcional sino de algo que el diario impulsaba activamente.

$2::$

www.theguardian.com/data

3::

Búsqueda del término "data journalism" en https://scholar.google. com/scholar (noviembre de 2015).

4::

El término "big data", o "datificación", refiere al hecho de que la tecnología permite transformar en datos una gran variedad de cosas, en orden de buscar, monitorear y optimizar prácticamente todo, desde la actividad física, la alimentación diaria o los procesos educativos. com/trends (17 de noviembre de 2015).

https://www.google. com/trends (19 de mayo de 2016). debates y reflexiones sobre el periodismo de datos (Martinho, 2014). Menos de dos años después se publicó el Manual del periodismo de datos, como producto de un taller efectuado en Londres en 2011. Docenas de periodistas de todo el mundo colaboraron en este manual, que es hoy el trabajo más citado sobre periodismo de datos, según Google Scholar.

El periodismo de datos es un hecho, pero continúa desarrollándose con la llamada big data o datificación $n^{4}$, mientras las salas de redacción siguen buscando nuevas formas de relatar historias en una época en que lo único permanente es el cambio. Una mirada al concepto de periodismo de datos en Google Trends ${ }^{5}$ - que muestra la cantidad relativa de búsquedas de ese término a través de los años- evidencia su auge desde 2010 y el creciente interés en esta forma de practicar el periodismo en la era digital. que se creó uno de los primeros documentos con

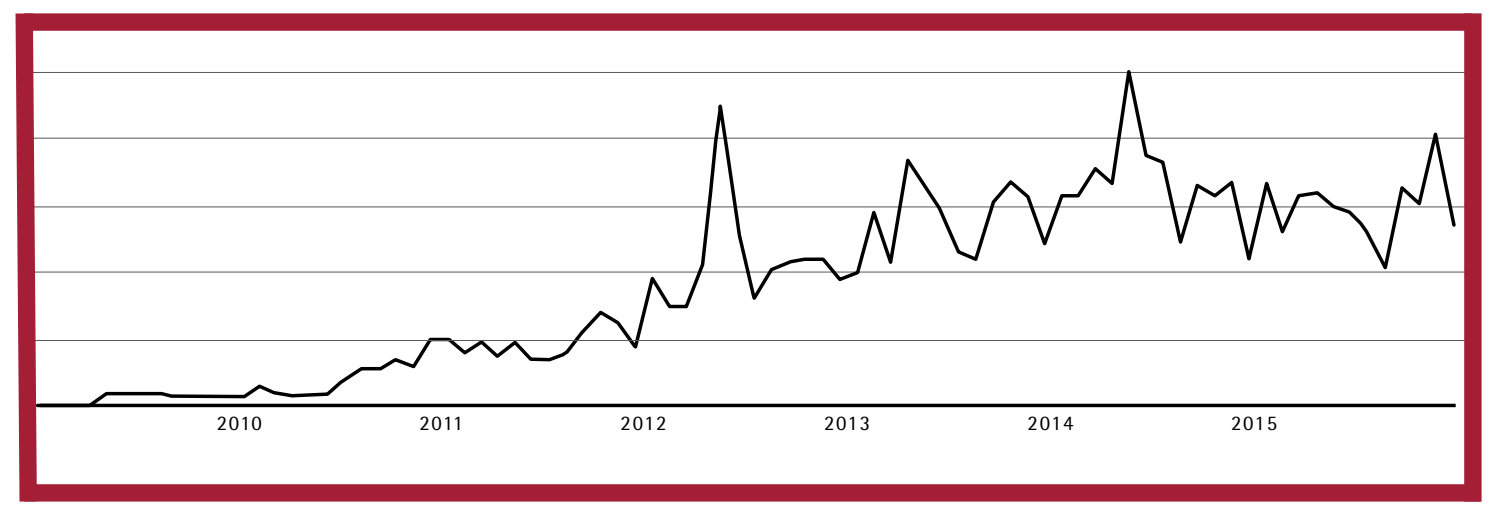

Número relativo de búsquedas del término “data journalism” en Google Trends ${ }^{6}$ 


\section{Periodismo de datos en los}

\section{medios uruguayos}

El espectro de medios de comunicación en Uruguay no es muy amplio. En la página web del Internet Advertising Bureau -formado por diferentes compañías afiliadas que tienen negocios en la red- figuran únicamente trece medios en Uruguay (IAB Uruguay, 2015). Y dentro de ese grupo, solamente ocho aparecen en las mediciones mensuales que establecen un estándar que garantiza a las agencias del medio y a los accionistas el tráfico en esas páginas. Este es el único parámetro digital que existe para los medios uruguayos presentes en la red, más allá de aquellos que cada medio realiza con sus propias herramientas.

Cuatro editores y directores de estos ocho medios completaron un cuestionario que investigaba si los recursos humanos de su medio estaban acostumbrados al periodismo de datos, cómo era el proceso actual en la sala de redacción y cómo habían sido las historias publicadas en los últimos 90 días, contados hasta principios de diciembre de $2015^{7}$.

Además, se realizaron dos entrevistas con preguntas abiertas para tener el punto de vista de jefes de redacción para ver cómo era su percepción sobre estado del periodismo de datos en el país, así como su visión del futuro de ese género y su desarrollo ${ }^{8}$.

\section{Recursos humanos}

Las redacciones de los medios todavía no han adaptado su lugar de trabajo para la actividad del periodismo de datos. Aunque todos los que completaron el formulario dijeron haber trabajado en proyectos de periodismo de datos, solo uno de ellos tiene un equipo establecido y en actividad (El Observador), dos tienen personal con cierta formación en periodismo de datos (Montevideo Portal y El Observador) y todos ellos usan los recursos que ya tienen para trabajar en este campo.

En los medios consultados parece existir una tendencia a tener periodistas trabajando en proyectos. Solamente uno (El Observador) dispone de una persona con dedicación total para trabajar noticias a partir de datos, mientras que los demás usan a los periodistas que ya tienen, así como diseñadores y programadores, para abordar algún proyecto. De esta manera surgen historias que en general no tienen información nueva, porque son historias convencionales mostradas de un modo diferente, más visual o interactivo.

Los principales autores de los proyectos del periodismo de datos -que son los mismos periodistas que están trabajando en otras tareas- no parecen estar debidamente preparados. Como dice Ana Laura Pérez, editora de El País Digital:

Los periodistas no están preparados para nada. Gestionar datos requiere conocimientos de matemáticas, estadísticas, Microsoft Excel, conocimientos que los periodistas uruguayos no manejan en absoluto y que ni siquiera están incluidos en los planes de las universidades donde se graduaron (A. L. Pérez, comunicación personal, noviembre 24, 2015).

Sin embargo, María Noel Domínguez, de Montevideo Portal, asegura que este tipo de competencias tienen cada vez más carga horaria en la academia (M. N. Domínguez, comunicación personal, noviembre 24, 2015).
7::

Los periodistas que llenaron el formulario fueron: María Noel Domínguez (editora de Montevideo Portal), José Frugoni (editor de Espectador.com), Carina Novarese (gerente de contenidos digitales de El Observador) y Martín Aguirre (director de El País).

$8::$

Las entrevistadas fueron María Noel Domínguez (editora de Montevideo Portal) y Ana Laura Pérez (editora de El País Digital). 
En lo que refiere a los recursos humanos, entonces, el periodismo de datos en Uruguay se parece a los portales digitales de noticias en el año 2000: todos saben que tienen que trabajar en eso, pero no tienen los recursos para hacerlo o no saben cómo hacerlo adecuadamente. Los periodistas hoy trabajan, de vez en cuando, en algunos proyectos de periodismo de datos sin esperar mayores resultados, porque no están verdaderamente comprometidos con el género; simplemente quieren explorar nuevas maneras de contar historias.

Se debe destacar un hecho interesante: la correlación entre el número de personas involucradas con las historias del periodismo de datos y el porcentaje de personas que trabajan para la página web del medio. Tomando como base ese dato, se puede afirmar que, para crecer, el periodismo de datos necesita en primer término más empuje en la sala de redacción digital. Sin embargo, esta afirmación debe ser confirmada por un mayor número de casos.

\section{El proceso del periodismo de datos}

En cuanto al proceso de elaborar noticias de periodismo de datos, las redacciones operan de modos muy diferentes. Algunas, como la de Montevideo Portal y la del Espectador.com, se concentran más en la creación de historias con atractivo visual, mientras que, por otro lado, El Observador y El País operan con un espectro más amplio, tanto en lo referente al modo de recoger los datos como en la manera de presentarlos.

En la mayoría de las redacciones, según declaran los encuestados, se trabaja con informaciones de instituciones privadas, como ONG o compañías encuestadoras. Y la mayoría también declara que trabaja con datos extraídos de instituciones públicas, ya sea con datos del dominio público o con datos recogidos en virtud de la ley de acceso a la información que existe en el país. Sin embargo, cuando se les pregunta si han trabajado con datos construidos por el propio medio, solamente la mitad responde afirmativamente.

Esto es coherente con lo que se dijo sobre la falta de especialización: para construir los datos no se necesita únicamente tiempo, sino también conocimiento de técnicas de investigación, metodología y estadística, áreas que la mayoría de los periodistas no conocen o no usan cuando escriben historias.

Aparentemente existe una contradicción entre lo que los medios consideran importante para una historia y el modo de elaborarla. Aunque la mayoría de los periodistas consultados concuerda en que lo más importante es que la historia tenga impacto (ver tabla), tanto las herramientas que usan como las historias que crean se centran en la visualización, en el modo de comunicación. En la mayoría de los casos se pone poco esfuerzo en las instancias de recoger datos (1) y en su análisis (2).

\begin{tabular}{|c|c|c|}
\hline \multicolumn{3}{|c|}{$\begin{array}{c}\text { Relevancia subjetiva de los aspectos de las } \\
\text { noticias de periodismo de datos } \\
\text { (ordenados de una lista dada) }\end{array}$} \\
\hline Aspecto & $\begin{array}{c}\text { Valor más } \\
\text { repetido }\end{array}$ & Promedio \\
\hline Impacto & 1 & 1.75 \\
\hline Atractivo visual & 2 & 1.75 \\
\hline Facilidad de lectura & 3 & 3.50 \\
\hline Interactividad & - & 3.50 \\
\hline Exclusividad & 5 & 4.50 \\
\hline
\end{tabular}


Entonces, puede decirse que actualmente en Uruguay se entiende por proceso del periodismo de datos la parte de organización y comunicación, mientras que los primeros pasos, recoger y analizar datos, se minimizan. Dos hechos apoyan esta afirmación: que ninguno de los periodistas ha trabajado con hackers y que la presencia de especialistas en economía o ciencias sociales es escasa o nula durante el proceso de elaboración de las historias. 0 sea que, aunque aceptan como hecho que la historia debe producir un impacto, no intervienen en las etapas del proceso donde se descubre el verdadero impacto de una historia: la recolección de nuevas informaciones y su análisis. No parecen concentrar sus esfuerzos en nuevas maneras de extraer datos y comprenderlos, sino más bien en las maneras de presentar la historia.

\section{Leyendo el futuro}

De acuerdo a los resultados de la encuesta, el factor más importante que interfiere en el desarrollo del periodismo de datos en las salas de redacción uruguayas es la falta de recursos humanos, seguido por la falta de tiempo y de apoyo de los directores del medio. No obstante, la mayoría de los consultados piensa que dentro de un año habrá más personas trabajando en este campo en las salas de redacción. En su opinión, eso no asegura que aumente el número de horas de trabajo, pero por lo menos señala que es posible.

Por otra parte, cuando se les pregunta de qué manera debería desarrollarse el periodismo de datos en sus redacciones, la mitad declara que no debería haber un aumento de trabajadores especializados sino un aumento de concientización y una mejora de las habilidades de las personas que ya están trabajando. De modo que el cambio hacia un periodismo de datos mejorado y más frecuente no vendría de abrir las puertas de las salas de redacción a personas nuevas, como los hackers, los sociólogos o los expertos en estadística, sino de formar a los periodistas que ya están en el medio.

Los diseñadores y los programadores, que hoy no son vistos como parte del equipo periodístico, probablemente tengan más que ofrecer al periodismo de datos de Uruguay que los propios periodistas. Como afırma Ana Laura Pérez:

Hay que aprender a trabajar con datos (cosa que la mayoría de los periodistas no manejan) y aprender a complementarse con diseñadores y programadores, y trabajar con ellos; no considerarlos elaboradores de accesorios decorativos (A. L. Pérez, comunicación personal, noviembre 24, 2015).

Una de las mayores amenazas para el periodismo de datos en Uruguay es el tamaño del mercado, que no permite a los medios hacer inversiones de alto riesgo. Especialmente en tiempos de desaceleración económica por la recesión brasileña y la debilidad argentina, producto del proteccionismo económico de los últimos años. Como dice Daniel Carranza, miembro de Data Uruguay -ONG que trabaja por la promoción de datos abiertos como herramienta de justicia social- el "presupuesto limitado" siempre es una buena excusa para inmovilizarse.

El periodismo de datos en Uruguay no despega. Hubo algunos esfuerzos aislados -algunos más serios que otros- pero ninguno tuvo continuidad. Las compañías de medios en Uruguay están en un círculo vicioso de 
caída-desinversión-caída, y en ese círculo cuesta mucho justificar los recursos de un tipo de periodismo que da resultados a mediano plazo. El periodismo de datos requiere fuertes inversiones en campos inexplorados para los medios (como los científicos de datos y los programadores). El periodismo de investigación viene sufriendo ese problema desde mucho antes y se agrava en un país con redacciones enteras de diarios que a veces son más chicas que el equipo de datos de un diario mediano europeo (D. Carranza, comunicación personal, diciembre 2, 2015).

\section{El periodismo de datos en la sociedad}

En Uruguay el periodismo de datos no está limitado a las corporaciones de medios o los medios de comunicación privados. En realidad, se han realizado vigorosos esfuerzos para alejarlo de ellos. Y no surgen solamente del sector público, sino también de esfuerzos realizados por las ONG, los centros de enseñanza y las personas que disponen de su tiempo libre para trabajar en proyectos de periodismo de datos.

\section{Datos abiertos y gobierno}

De acuerdo al Índice Oficial de Datos de 2015, Uruguay está en la séptima posición en la escala global de los gobiernos de datos abiertos (Open Knowledge, 2015). En los últimos años, el Parlamento aprobó la Ley de Acceso a la Información Pública (LAIP) y se creó una oficina llamada AGESIC para impulsar la apertura gubernamental y para incentivar el uso de soluciones electrónicas para mejorar la democracia. A pesar de eso, la aplicación de la ley todavía encuentra obstáculos que impiden alcanzar el ideal de un gobierno verdaderamente abierto.
La Ley de Acceso a la Información Pública fue aprobada en el año 2008 como consecuencia del trabajo de activistas de la sociedad civil, así como de parlamentarios que vieron la necesidad de mejorar la calidad de la gestión pública (Fernández, 2014). La ley establece como objetivo promover la transparencia de la acción gubernamental en todas las instituciones públicas, y garantizar el derecho de los ciudadanos a acceder a la información pública. También dice que cualquier persona tiene ese derecho, por tanto, no es necesario presentar una justificación ni especificar el nombre o la nacionalidad cuando se solicita una información con amparo en esta ley (Poder Legislativo, 2008).

No obstante, tres de los cuatro periodistas consultados en la encuesta afirmaron que su medio había solicitado información pública y que, en todos los casos, su solicitud había sido rechazada por la oficina pública. La LAIP establece siete casos de excepción en los cuales el Estado puede negarse a dar información pública; por ejemplo, si se trata de la seguridad nacional o de información que puede causar daño a un particular si se divulga. Si la oficina no responde en veinte días hábiles o si el solicitante considera que la cláusula de excepción no es válida para su caso, puede entablar una demanda para que la Justicia decida si la información debe darse o no.

Pero la ley, cuyas intenciones parecen estar tan claramente formuladas, choca de frente con la realidad. Un estudio de la Fundación Friedrich-Ebert-Stiftung (FES) realizado en 2014 mostró que, tomando en consideración distintas variables, de 130 solicitudes de información presentadas en oficinas públicas, menos del $20 \%$ fueron respondidas positivamente (Piñeiro Rodríguez y Rossel, 2014). Esto concuerda con el 
índice ITAeL, calculado todos los años por CAInfo y la Universidad Católica del Uruguay, que mide los niveles de transparencia activa del gobierno nacional y los gobiernos departamentales. En 2014 el índice promedio en los ministerios fue de $41 \%$, mientras que para los gobiernos departamentales fue de 35\% (Ponce, Santángelo y Pérez, 2015). Este bajo nivel de cumplimiento de la ley puede no responder solamente a ignorancia de la LAIP sino también a lo que se ha dado en llamar "cultura del silencio" en las dependencias del Estado, expresión que alude al hecho de que muchas veces los empleados públicos prefieren callar antes que decir algo que les pueda traer problemas.

El índice ITAeL también muestra que el nivel de transparencia fluctúa mucho de oficina en oficina, y que falta mucho para convertirlo en realidad. Daniel Carranza, de Data Uruguay, afirma que así se explica que hoy en día AGESIC y UAIP, las agencias gubernamentales responsables del gobierno abierto y de la transparencia, tengan tanto que hacer (D. Carranza, comunicación personal, 2 de diciembre de 2015). "Sin duda la LAIP es positiva, pero su implementación no ha sido tan buena como se esperaba", dice Manuel Podetti, de Hacks/Hackers (M. Podetti, comunicación personal, diciembre 4, 2015).

El apoyo estatal y las iniciativas de las ONG Aparte de la LAIP, el gobierno tuvo otras iniciativas para impulsar el periodismo de datos en el país. En realidad, la mayoría de los proyectos han estado a cargo de múltiples actores, y en muchos de ellos las empresas de medios no han estado siquiera presentes. La variedad de iniciativas muestra hasta qué punto el crecimiento del periodismo de datos ha dependido del interés personal de algunos profesionales, con el apoyo técnico y financiero de organizaciones privadas y entes públicos como AGESIC.

Manuel Podetti es integrante de Hacks/Hackers Uruguay, representante local de una organización con presencia en más de 60 ciudades, cuyo objetivo principal es reunir diferentes profesionales tales como periodistas (hacks) con diseñadores y programadores (hackers) para colaborar en proyectos periodísticos como gráficos interactivos, infografías, mapas y cronogramas. Completan el equipo otros integrantes: Gabriel Cicarello, Federico Comesaña y Jimena Abad, de El Observador y la Universidad Católica del Uruguay. En 2014 y 2015 organizaron dos DataCamps, ${ }^{9}$ con el apoyo de AGESIC. Fueron eventos de tres dias de duración en los que los participantes, luego de asistir a una serie de charlas sobre periodismo de datos, trabajaron en equipo para conseguir historias periodísticas elaboradas por ellos mismos en el campamento.

De iniciativas similares se ocupa la organización Data Uruguay. Según Daniel Carranza, las organizaciones sociales comenzaron a trabajar en el periodismo de datos antes que los medios. En su página web, Data Uruguay exhibe muchos de sus proyectos, que en su mayoría fueron realizados en colaboración con medios, otras ONG u organismos públicos. Algunos incluso ganaron premios, como A tu servicio, una página creada por el Ministerio de Salud Pública que informaba sobre la calidad de la asistencia en las distintas proveedoras de salud del país (Data Uruguay, s. f.).

Las ONG trabajan con datos y saben procesarlos y mostrarlos, existe un enorme potencial de colaboración y beneficio mutuo con los medios. Sin embargo, ese potencial
9::

Jornadas de entrenamiento en datos. 
existe desde prácticamente siempre, no se limita al periodismo de datos. Los desafíos son las formas de colaboración, alinear los incentivos de exclusividad de un medio con el de máxima difusión de una ONG y los modelos de negocio que permitan que colaboraciones así consigan fondos. El caso más interesante es probablemente UYCheck, que viene haciendo periodismo de datos de forma continua desde hace un año y no es un medio, es una ONG. (D. Carranza, comunicación personal, diciembre 2, 2015).

UYCheck, ${ }^{10}$ fundada por estudiantes de Ciencias Políticas, averigua si la información brindada por políticos o figuras públicas es cierta o no ("UYCheck, un sitio de chequeo para una ciudadanía 'más crítica”, 2014). Esta página web comenzó a cobrar importancia en la política uruguaya durante la última campaña electoral. Después de asumir funciones el nuevo gobierno, en marzo de 2015, UYCheck lanzó www.deldichoalhecho.com.uy, una página donde se controla cuántas de las promesas hechas por el presidente y su partido durante la campaña electoral se están cumpliendo. La web se construyó con el apoyo de una organización chilena llamada Ciudadano Inteligente, que ha estado manejando una página similar en Chile en los últimos años.

\section{La educación y el periodismo de datos}

En muchos casos, las universidades que trabajan en el campo de las Comunicaciones también han participado en los esfuerzos de una sociedad solidaria para impulsar el periodismo de datos. En la página de reuniones de Hacks/Hackers Uruguay, por ejemplo, el único patrocinador que se muestra es la Universidad Católica del Uruguay, lugar donde algunos de los miembros de la organiza-

$$
\text { ción dan clases sobre periodismo de datos a los }
$$
futuros periodistas.
Jimena Abad, docente de la asignatura Periodismo en Internet en la Universidad Católica del Uruguay, relata que el periodismo de datos ingresó en la universidad por primera vez en el año 2013, como un tema más dentro del programa de estudios (J. Abad, comunicación personal, diciembre 12, 2015).

En el 2014 algunos especialistas fueron invitados a la Universidad a hablar sobre eso y se realizó la primera hackatón, una jornada especial dedicada al periodismo de datos. Pero, en 2015, Abad y sus colegas de El Observador, Federico Comesaña y Gabriel Cicarello, presentaron un proyecto que fue aprobado por la universidad: consistía en tres clases obligatorias de periodismo de datos para los estudiantes, charlas con los especialistas y dos hackatones que se realizarian en el segundo semestre del año.

El proceso de integrar el periodismo de datos al centro de enseñanza sigue avanzando, y Abad espera que crezca aún más dentro de la Universidad. El impulso que le imprimió a esta iniciativa fue producto de su propia frustración cuando trató de implementar técnicas de periodismo de datos en la sala de redacción donde trabajaba en ese momento:

Junto a Federico y Gabriel intentamos hace un par de años impulsar en El Observador (donde trabajaba) la creación de un equipo de periodismo de datos, pero no fue viable. Entonces, en mi opinión, la forma de impulsar el periodismo de datos era dar vuelta la ecuación y enseñar a los estudiantes -que mañana se insertarán en las redaccionesherramientas para que usen el periodismo de datos como una fuente más y mejoren la calidad de la información que damos en Uruguay (J. Abad, comunicación personal, diciembre 12, 2015). 
En la Universidad de la República el periodismo de datos ha aparecido de la misma manera, como un esfuerzo aislado, y sigue luchando contra los obstáculos que surgen en su camino. Betania Núñez, profesora del Taller de periodismo de la Facultad de Información y Comunicación, diseñó en 2015 un curso llamado Técnicas digitales para el periodismo, en el que el periodismo de datos ocupa dos tercios del programa. Este curso es, según dice, un gran desafío en tres aspectos: primero, requiere una formación constante de los profesores; segundo, la mayor parte de los alumnos no saben nada de periodismo de datos antes de asistir al curso; y tercero, las propias herramientas disponibles en la Universidad representan en sí un desafío especial, porque no siempre están en buenas condiciones (B. Núñez, comunicación personal, noviembre 28,2015$)$.

Núñez afırma que lo primero que deben hacer las universidades para desarrollar el periodismo de datos es incorporarlo al plan de estudios de los futuros periodistas. No solamente como asignatura, sino como tema de talleres. También debieran insertarlo en los trabajos prácticos y coordinar mejor la docencia para incorporarlo como destreza para los estudiantes (Núñez, comunicación personal, noviembre 28, 2015). Esto concuerda con lo que se dice en los medios: que los periodistas hoy no tienen los conocimientos necesarios para elaborar historias de periodismo de datos y que todos los miembros de la sala de redacción deberían estar capacitados en ese sentido, que los especialistas en periodismo de datos no debieran ser los únicos en poseer esas destrezas.

Álvaro Pérez, coordinador del área de Periodismo en la Universidad de Montevideo, expresa lo siguiente:
El escenario ha cambiado, y si los periodistas son capaces de diseñar webs y tener otros conocimientos, ganamos todos. Un técnico puede hacer una página web muy bonita desde el punto de vista estético, pero lo importante es que se lea bien, que la información esté bien organizada. Ocurre lo mismo que cuando se diseña una página de diario: el diseño debe estar al servicio de la información. Lo que quiero decir es que el periodista, antes de nada, deber ser buen periodista, y las personas que están alrededor, si no son periodistas, al menos deben trabajar con periodistas (A. Pérez, comunicación personal, noviembre 24, 2015).

Todos los entrevistados en las universidades están de acuerdo en reconocer que, a pesar de los nuevos desafíos que ofrece la tecnología (como el que representa el periodismo de datos), la esencia del buen periodismo va más allá del uso de herramientas especiales o de géneros específicos. En ese sentido, Álvaro Pérez -en cuya universidad el periodismo de datos no está presente más que en la voluntad de los estudiantes de realizar ese tipo de proyectos y en el uso de ejemplos en las lecciones de Periodismo Escrito-declara:

El problema de las instituciones educativas es que el periodismo real va más rápido que el "teórico". No es que un académico inventara el género "crónica" y los periodistas comenzaran a usarlo, sino al revés. Considero que las universidades deben, en primer lugar, hacer hincapié en lo que permanece y es consustancial al periodismo, independientemente del formato. Y, en segundo lugar, no ser ajenos a estos 
cambios, tratar de incorporarlos y estudiar cómo pueden aprovecharse como aliados de la profesión y no como enemigos (A. Pérez, comunicación personal, noviembre 24, 2015).

Las universidades hoy consideran entonces que el periodismo de datos es algo que merece no ser ignorado. Algunas instituciones despliegan más esfuerzos trabajando en ese tipo de periodismo con sus estudiantes, mientras que otras están en una fase analítica y esperan ver cómo resulta en el futuro para decidir si vale la pena integrarlo formalmente o no al plan de estudios. Pero todas las universidades reconocen su existencia, y asumen el papel que juegan como educadoras de los futuros periodistas del país.

\section{Conclusión}

A través de los años, el esfuerzo por impulsar el periodismo de datos en Uruguay se ha ido intensificando. No obstante, algunos actores clave, como los medios privados, todavía no quieren correr los riesgos económicos necesarios para desarrollar un trabajo profesional y serio en esta área. Mientras las $\mathrm{ONG}$, los organismos públicos y los centros de enseñanza se esfuerzan reiteradamente, los medios privados aún no reaccionan porque la inversión aparenta ser poco redituable en el corto plazo.

Las organizaciones y las ONG, en especial, están trabajando más que nunca para que los medios reaccionen, pero ante su falta de respuesta están enfocando sus esfuerzos cada vez más hacia sus propios proyectos y a proyectos de particulares o grupos que no tienen un interés económico en ese tipo de periodismo, como otras ONG y proyectos creados por personas interesadas por esa área.

El gobierno, por su lado, lucha para llegar a mejores niveles de transparencia, ayudado por las investigaciones de las ONG. Aunque el nivel de transparencia del Uruguay es bueno comparado con el de otros países, la realidad está alejada del ideal, porque la Ley de Acceso a la Información no está funcionando de acuerdo a lo esperado y las oficinas del Estado se defienden guardando sus secretos.

Mientras tanto, las universidades, habiendo observado el crecimiento del periodismo de datos, están tratando de introducirlo en sus programas, pero sin precipitarse y ofreciéndolo más como una destreza o una opción para sus estudiantes de periodismo. Se trata de una destreza demandada por medios que quieren empezar a trabajar en periodismo de datos, pero que no disponen de los recursos ni de los conocimientos para un emprendimiento serio y tampoco pueden ni quieren hacer inversiones de riesgo.

La cuestión es si los medios van a ponerse a la par de los esfuerzos de los otros actores para hacer del periodismo de datos una realidad en el Uruguay o si, como sucedió con el periodismo de investigación, los esfuerzos de las ONG, las universidades y los particulares se irán desvaneciendo lentamente.

\section{Referencias}

British Broadcasting Corporation (2011, octubre 27). The world at seven billion. Recuperado de http://www.bbc. co.uk/news/world-15391515

Bounegru, L., Lorenz, M., Rütten, W. (2010). Data-driven journalism: What is there to learn? Hudson, H. (Ed.) A paper on the data-driven journalism roundtable held in Amsterdam on 24 August 2010( p. 78). Ámsterdam: EJC.

Dader, J. L., Gómez, P. (1993). "Periodismo de Precisión»: una nueva metodología para transformar el periodismo. Anàlisi, 15, 99-116.

Data Uruguay (s. f.). A Tu Servicio. Recuperado de http://www. datauy.org/portfolio/a-tu-servicio 
Fernández, M. (2014). Acceso a la Información Pública en Uruguay. Perry, F., Paz, M. (Eds.). Manual de Periodismo de Datos Iberoamericano. Recuperado de http://manual. periodismodedatos.org

Gray, J., Bounegru, L., Chambers, L. (2012). The Data Journalism Handbook. Recuperado de http://datajournalismhandbook.org

IAB Uruguay (2015). Socios. Recuperado de http://www.iab. com.uy/socios/

Kuutti, T., Heikki, U. (2015). Models and streams of data Journalism. The Journal of Media Innovations, 2 (1), 77-88.

Lorenz, M. (2010, junio). Data-driven journalism: What is there to learn? Stanford: IJ-7. Recuperado de http:/| www.slideshare.net/mirkolorenz/datadriven-journalismwhat-is-there-to-learn

Martinho, A. P. (2014). Jornalismo de dados: caraterização e fluxos de trabalho. Exedra, 9, 64-73.

Meyer, P. (1991). The New Precision Journalism. Recuperado de http://www.unc.edu/ pmeyer/book

Open Knowledge (2015). Uruguay is ranked \#7 in the 2015 Index. Recuperado el 18 de diciembre, 2015, de http:// index.okfn.org/place/uruguay

Piñeiro Rodríguez, R., Rossel, C. (2014). Del dicho al hecho:derecho de acceso a la información pública en Uruguay. Recuperado de http://library.fes.de/pdf-files/bueros/uruguay/11587.pdf
Poder Legislativo (2008, noviembre 7). Ley 18.381. Recuperado de https://parlamento.gub.uy/documentosyleyes

Ponce, M., Santángelo, G., Pérez, J. (2015). Índice de Transparencia Activa en Línea 2014. CAlnfo, Universidad Católica del Uruguay. Recuperado de http:// itael.cainfo.org.uy/wp-content/uploads/2015/09/\%C3\%8Dndice-de-Transparencia-Activa-En-L\%C3\%ADnea-20140k.pdf

Rogers, S. (2011, setiembre 26). The first Guardian data journalism: May 5, 1821. The Guardian. Recuperado de http://www.theguardian.com/news/datablog/2011/ sep/26/data-journalism-guardian

Träsel, M. (2014). Historia del periodismo de datos en Brasil. Perry, F., Paz, M. (Eds.). Manual de Periodismo de Datos Iberoamericano. Recuperado de http:// manual.periodismodedatos.org

UyCheck, un sitio de chequeo para una ciudadanía "más crítica." (2014, octubre 17). El Observador. Recuperado de http://www.elobservador.com.uy/uycheck-un-sitio-chequeo-una-ciudadania-mas-critica-n290162

Yagoda, B. (2014, marzo 6). A short history of "hack". The New Yorker. Recuperado de http://www.newyorker. com/tech/elements/a-short-history-of-hack 